\section{A life or death situation}

The unfolded protein response (UPR) is triggered by the detrimental accumulation of unfolded proteins in the endoplasmic reticulum. UPR activates cytoprotective pathways that reduce translation and induce chaperone expression to help rescue protein folding, but it also induces proapoptotic mechanisms. To elucidate how these paradoxical responses are resolved, Lin and colleagues have followed the induction of these three distinct UPR pathways, mediated by IRE1, ATF6, and PERK, over time upon exposure to protein misfolding agents. Although IRE1-responsive events as well as ATF6 induction are triggered but then attenuate, proapoptotic responses are maintained over a longer period. To test the idea that attenuation of the former pathway is key to the onset of cell death, the authors created an IRE1 mutant whose activity can be selectively maintained by the small molecule 1NM-PP1. Cell survival increased when IRE1 activity was thus maintained, even after prolonged exposure to UPR-inducing drugs. The authors then examined a misfolding rhodopsin mutant that is associated with a form of retinitis pigmentosa, showing that it induces the UPR in the absence of drugs. Expression of this mutant in the rodent retina indicates that proapoptotic markers are induced and maintained, whereas a cytoprotective marker is eventually attenuated, correlating with the onset of retinal cell death. Although future work will be needed to clarify the mechanism of temporal UPR control, and how the apoptotic response is initially induced but prevented from taking hold, the findings provide a molecular framework for testing how the cell first tries to salvage the situation, before taking the step of eliminating itself. (Science 318, 944-949, 2007)

\section{$S L$}

\section{Getting loaded}

In eukaryotic cells, DNA replication initiates from numerous origins and must be coordinated such that each origin is activated only once during each $\mathrm{S}$ phase. To this end, a system has evolved that features periodic assembly and disassembly of essential pre-replicative complexes (pre-RCs) at replication origins. This process involves the loading of the $\mathrm{Mcm} 2-7$ helicase by the origin recognition complex (ORC) and two essential factors, Cdc6 and Cdt1. To study the function of Orc6, the smallest subunit of the six protein complex and the only one that is unrelated to AAA+ ATPases, Bell and coworkers used a combination of in vivo and in vitro analyses during the Saccharomyces cerevisiae cell cycle. They showed that Orc6 is not required for ORC DNA binding in vivo but is essential for assembly and maintenance of pre-RCs. In vitro studies revealed that Orc6 is required for $\mathrm{Cdt} 1$ and $\mathrm{Mcm} 2-7$, but not $\mathrm{Cdc} 6$, association with origin DNA and that this function can be bypassed by tethering full-length Cdt1 to ORC lacking most of Orc6. In addition, experiments with ORC $\Delta 6$ tethered to Cdt 1 support a model in which Cdt 1 binding and release are important for the loading of multiple $\mathrm{Mcm}$ 2-7 complexes at the origin. Although Ocr6 is the least conserved of the ORC subunits, it is possible that Orc6 and Ctd 1 have coevolved to maintain their interactions. Further work will be required to determine whether the dynamic association between Cdt1 and ORC is required for repeated $\mathrm{Mcm}$ 2-7 loading in other organisms. (Genes Dev. 21, 2897-2907, 2007)

$B K$

Written by Boyana Konforti, Sabbi Lall \& Michelle Montoya

\section{Stalling for time}

Transcription by RNA polymerase II (Pol II) takes place in a stepwise manner, with initiation complex formation followed by promoter escape and elongation. However, Pol II stalling has also been observed, immediately downstream of the transcription start site. To determine where Pol II stalling occurs during development in flies, Zeitlinger et al. analyzed global Pol II occupancy in whole Drosophila embryos. 76\% of genes could be assigned to one of three distinct classes: those where Pol II was distributed throughout the transcription unit (27\%), those where Pol II was enriched at the transcription site (12\%) and those that lacked Pol II binding altogether (37\%). Among the genes with stalled Pol II, $62 \%$ had Pol II tightly restricted to the transcription start site. The genes with stalled Pol II were highly enriched for genes important in neurogenesis, ectoderm development and muscle differentiation. The set of genes with uniform Pol II binding was, however, enriched for ubiquitously expressed genes involved in metabolism and cell proliferation. The genes lacking Pol II were highly enriched for those previously shown to have no early embryonic expression. Pol II stalling could reflect transcriptional repression or it could represent a state in which the gene is poised for activation at later stages of embryogenesis. In this study, the authors found evidence for both models, and they propose that Pol II stalling promotes rapid temporal and spatial changes in gene activity in response to developmental signals during embryogenesis and thus may represent a key regulatory for gene regulation in development. (Nat. Genet. published online 11 November 2007, doi:10.1038/ng.2007.26)

$B K$

\section{Flipping a growth switch}

Polarized membrane growth occurs during the early stages of yeast budding, when the bud undergoes apical growth followed by an isotropic phase, resulting in a bud with an ellipsoid shape. The switch in growth phase is cell-cycle regulated, with delays in switching resulting in elongated cells. When cytokinesis occurs, the growth site is polarized at the bud neck. The small GTPase Cdc42p coordinates polarized membrane growth by directing actin cytoskeleton regulators to growth sites. Interestingly, deletions of Lem3p, Dnf1p, and Dnf2p-proteins implicated in phospholipid flipping across bilayers, or 'flippases' - also produce cells with elongated shapes typical of excess apical growth. Tanaka and colleagues now show that lem $3 \Delta$ cells have excessive localization of Cdc42p at the bud tip. Overexpression of the Cdc42p-specific GTPase activating proteins (GAPs) Rgalp and Rga2p rescued the hyperpolarized growth phenotype and restored normal localization patterns of $\mathrm{Cdc} 42 \mathrm{p}$ during the budding cycle. Phosphatidylethanolamine (PE) is normally enriched in the membrane inner leaflet, being present at the outer leaflet only at polarized sites during the early stages of budding. In lem $3 \Delta$ cells, the outer leaflet of the bud tips was enriched in $\mathrm{PE}$, suggesting a loss of the ability to translocate PE inward. In vitro assays showed that the GAP activity of Rgalp and Rga2 $p$ were enhanced by PE and phosphatidylserine but inhibited by $\mathrm{PI}(4,5) \mathrm{P}_{2}$. In all, the findings suggest that the flippase activity of Lem3p and associated proteins is required for the switch from apical to isotropic growth. Although the downstream effects of the Lem3p-related activity seem to be fleshed out, more work is needed to figure out how cell-cycle regulators communicate these changes to the membrane. (Dev. Cell 13, 743-751, 2007) 